\title{
The Potential Role of Vitamin B12 in the Prevention of COVID-19 Complications: A Narrative Review
}

\author{
Srinidhi Rai ${ }^{1 *}$ (D), Sindhu², Prajna ${ }^{3}$, B. Shamantha Rai ${ }^{4}$ iD and P. Rithesh Pakkala ${ }^{4}$ \\ ${ }^{1}$ Department of Biochemistry, KS Hegde Medical Academy, Nitte (Deemed to be University), \\ Mangaluru - 575 018, Karnataka, India. \\ ${ }^{2}$ Department of Biochemistry, KMC Mangalore (Manipal Academy of Higher Education), Mangaluru - 575001 , \\ Karnataka, India. \\ ${ }^{3}$ Department of Biochemistry, Chamarajanagar Institute of Medical Sciences, Yadapura - 573 313, Karnataka, \\ India. \\ ${ }^{4}$ Department of Information Science \& Engineering, Sahyadri College of Engineering \& Management, \\ Mangaluru - 575007, Karnataka and Affiliated to Visvesvaraya Technological University, Belagavi, Karnataka, \\ India.
}

\begin{abstract}
The elderly are more prone to mortality from COVID-19 infection, as they are susceptible to develop acute respiratory distress syndrome (ARDS). For COVID-19 patients with ARDS caused by sepsis or septic shock, high-dose parenteral vitamin B12 appears to be a potential new treatment option. Vitamin B12 may play a substantial impact in COVID-19 morbidity and mortality reduction owing to its function in DNA synthesis, cellular control, improvement of anti-inflammatory immune responses, and decrease of pro-inflammatory responses. This review aims to assess the functional role of Vitamin B12 in COVID-19 in terms of its immunomodulatory effect, role in cellular and humoral immunity and maintaining the gut microbe homeostasis. From data inception to June 2021, accessible electronic databases were searched for research/review articles reporting on the function of Vitamin B12 in COVID-19. Scopus, Web of Science, PubMed, WHO worldwide research on COVID-19 and the clinical trials registration "https://clinicaltrials.gov/" were used to conduct the systematic search by using keywords: "COVID-19 and "Vitamin B12". Also, based on these outcomes, it can be concluded that Vitamin B12 may have a potential role in preventing COVID-19 complications. Further, studies evaluating the role of Vitamin B12 in COVID-19 may open a new array of ideas on the optimal and the well-tolerated dose and timing of its administration in COVID-19 patients.
\end{abstract}

Keywords: Vitamin B12, COVID-19 Complications, Acute Respiratory Distress Syndrome, Mortality Reduction, Immunomodulatory Effect

*Correspondence: srinidhirai@nitte.edu.in; +919591247186

(Received: August 20, 2021; accepted: September 29, 2021)

Citation: Rai S, Sindhu, Prajna, Rai BS, Pakkala PR. The Potential Role of Vitamin B12 in the Prevention of COVID-19 Complications: A Narrative Review. J Pure Appl Microbiol. 2021;15(4):1735-1744. doi: 10.22207/JPAM.15.4.04

(C) The Author(s) 2021. Open Access. This article is distributed under the terms of the Creative Commons Attribution 4.0 International License which permits unrestricted use, sharing, distribution, and reproduction in any medium, provided you give appropriate credit to the original author(s) and the source, provide a link to the Creative Commons license, and indicate if changes were made. 


\section{INTRODUCTION}

SARS-CoV-2 is a virus with a positivesense single-stranded RNA genome that is the causative agent of COVID -19, which was initially detected in Wuhan, China in 2019 and has since caused countless deaths and economic losses all over the world. ${ }^{1}$ As of August 2021, the number of active cases of COVID-19 in India was $1.21 \%^{2}$ showing a significant increase in the number of new cases in many states of India, raising the possibility of a third wave of COVID-19, following the end of a devastating second wave of COVID-19. The COVID-19 pandemic has a particularly negative impact on the elderly and people with type 2 diabetes, imposing greater inpatient treatment and a higher death rate..$^{3,4}$ COVID-19 problems in the elderly and diabetics are mostly caused by reduced glucose tolerance, aberrant T-cell responses, underlying comorbidities linked to low-grade inflammation, and viral entry factors. ${ }^{5}$ Mutations has been occurred in the SARS CoV-2 virus throughout time. The presently available SARS CoV-2 vaccine offers some protection against new versions. COVID-19, however, needs a continual concern due to mutations, the possibility

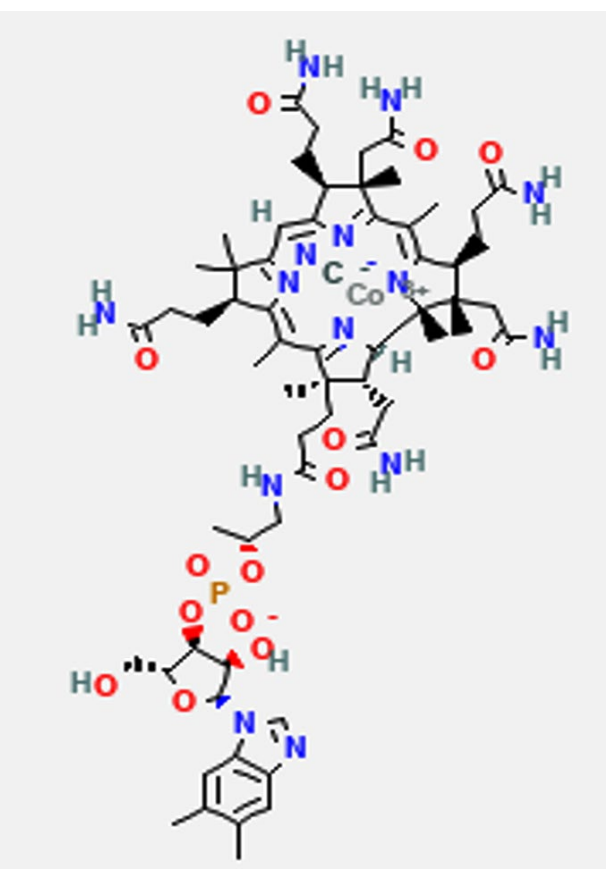

Fig. 1. Structure of vitamin B12, retrieved from PubChem Compound Database, National Center for Biotechnology. ${ }^{8}$ of recurrent infection, infectivity, and a wide variety of clinical manifestations, particularly among the elderly and diabetic patients, who are predominantly vulnerable. ${ }^{6}$ To determine the impact of diet on COVID-19, more than 40 research studies have been reported. The majority of them have focused on COVID-19 and the effects of vitamin $D$, vitamin $C$, and the mineral zinc. ${ }^{7}$ However, there is currently no clear evidence on the effects of vitamin B12 deficiency. This narrative review summarizes the current knowledge about the structure, kinetics and functions of vitamin B12. It also describes the mechanistic reason behind the possible protective effect of Vitamin B12 in COVID-19 complications and the evidence with regard to vitamin B12 supplementation. It's extremely similar to how COVID-19 causes harm: immunologically, microbiologically, hematological, and through endothelial cell signaling-supporting the theory that B12 deficiency is a possible COVID-19 risk factor.

\section{Structure and Kinetics of Vitamin B12}

Vitamin B12 (Cobalamin) is a complex organometallic compound with a centrally placed cobalt atom (Fig. 1). Vitamin B12 has four pyrrole rings that are comparable to porphyrin. At the center of the corrin nucleus, the four nitrogens of the tetrapyrole structure are bound to a cobalt atom. The cobalt-corrin complex is known as cobamide.

Four of the six coordination sites are provided by corrin ring and benzimidazole provides the fifth group. The sixth coordination site is provided by a cyano group $(-\mathrm{CN})$, a hydroxyl group $(-\mathrm{OH})$, a methyl group $(-\mathrm{CH} 3)$ or a $5^{\prime}$-deoxyadenosyl group. It is synthesised only by microbial synthesis of the digestive tract.

Absorption of vitamin B12 occurs in the terminal ileum with the help of two binding proteins. The gastric parietal cells produce the intrinsic factor of castle (IF), a glycoprotein. Cobalophilin, a salivary protein, also plays an important role in absorption of vitamin B12. Vitamin B12 is released from dietary protein via pepsin, which then binds to a protein called cobalophilin. Trypsin from pancreatic juice hydrolyzes the cobalophilin, releasing the vitamin, which then attaches to the castle's intrinsic factor. Two molecules of vitamin B12 can bind to one molecule of intrinsic factor. The IF-B12 complex 
binds to particular receptors on mucosal cells. ${ }^{9}$ Vitamin B12 absorption declines as people age. As a result, deficiency is prevalent among the elderly, those who have had a gastrectomy, ileal resection, and malabsorption disorders. Vitamin B12's active coenzyme forms, methyl cobalamin and adenosyl cobalamin, influence its activity. ${ }^{10}$

\section{Functions of Vitamin B12}

Hydroxo-, adenosyl-, and methyl cobalamin are the active forms of vitamin B12. Vitamin B12 is a gut microbiota modulator, and low levels of B12 raise methyl malonic acid and homocysteine, leading to increased inflammation, reactive oxygen species, and oxidative stress. ${ }^{11}$ Vitamin B12 has recently been discovered to have an important part in the regular functioning of the brain and neurological system, as well as the production of blood. Vitamin B12 is required for a variety of metabolic processes, including DNA synthesis and control, fatty acid synthesis, and energy generation. ${ }^{12}$

The active form of vitamin B12, methyl cobalamin, aids in the production of methionine and S-adenosyl methionine. Myelin integrity, neurological function, synthesis of red blood cell and DNA synthesis are all dependent on it. Vitamin B12 deficiency is most common in vegetarians across the world, and it may be treated with Methyl cobalamin, a vitamin B12 analogue. ${ }^{13}$ Methyl cobalamin serves as a coenzyme in the transfer of methyl group from N5 methyl tetrahydrofolate to homocysteine for the formation of methionine (Fig. 2).

Adenosyl cobalamin, also known as coenzyme-B12, is another biologically active form of vitamin B12. ${ }^{14}$ The cofactor's role as an initiator of the reactive free radicals required for these reactions is well understood. How these

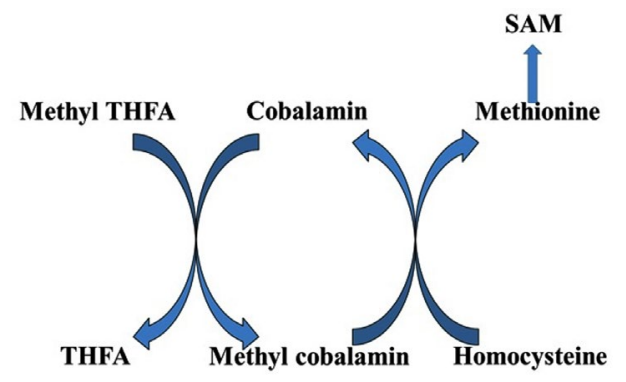

Fig. 2. Role of Methyl Cobalamin enzymes drive the coenzyme towards hemolysis and regulate the radicals that are produced is less known. Enzymes have evolved a variety of methods for producing reactive radical species that may be utilised to activate $\mathrm{C}-\mathrm{H}$ bonds. It's worth noting the strong molecular similarities between AdoCbl-dependent enzymes and the considerably broader family of radical S-adenosyl methionine (SAM) enzymes, which was recently discovered (Fig. 3). ${ }^{15}$ Several adenosyl cobalamin enzymes have been computationally modelled in some detail made to the availability of high-resolution $X$-ray structures paired with comprehensive kinetic and spectroscopic studies. Computer models have typically agreed well with experimental data and have revealed new information about the processes behind these unexpected reactions. ${ }^{15}$

Nutrition plays a major role in providing immunity against COVID -19 infection which may be responsible for the differences in the severity of COVID -19 infection seen in different parts of Europe. ${ }^{16}$ Countries who have reported a lower death rate largely consumed diet that is rich in antiangiotensin converting enzyme activity (anti-ACE) and antioxidant activity, for example, cabbage. ${ }^{17-19}$ Nutrition plays a very important role during COVID-19 pandemic and its significance is to be better understood. Therefore, this review aims to assess the role of Vitamin B12 as a potential modifiable risk factor in COVID-19.

\section{Brief Literature Search}

This narrative review highlights a brief non-systematic methodology to include the

\section{Propionyl CoA}

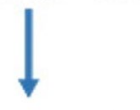

D methyl malonyl CoA

\section{L methyl malonyl CoA}

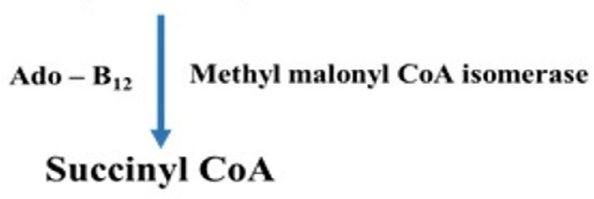

Fig. 3. Role of Adenosyl Cobalamin. 
selected articles for discussion. To evaluate the current knowledge and understanding of relationship between Vitamin B12 and COVID-19, an online literature search on Scopus, Web of science, Pubmed, WHO global research on COVID-19 and the clinical trials register "https:// clinicaltrials.gov/" data bases was done from data inception to June 2021 using the following MeSH terms (Corona virus disease 2019 or COVID-19), SARS- CoV-2, Vitamin B12 and Vitamin B12 deficiency. Only published original and review articles in English were included. Articles which included paediatric age and pregnant women were excluded.

The experimental area, methodology adopted and outcome of the studies which assessed Vitamin B12 levels in COVID-19 patients are illustrated in Table 1.

Role of Vitamin B12 In Mediating The Inflammatory Response

Vitamin B12 enhances the number of lymphocytes including $\mathrm{CD} 8^{+}$cells and natural killer cells thus playing a pivotal role in mediating immune function, mainly on cytotoxic cells. It prevents apoptosis of CD $8^{+}$cells. ${ }^{22}$ Invitro studies have shown that vitamin B12 was involved in concanavalin a dependent T-cell production and pokeweed mitogen dependent immunoglobulin synthesis in B cells. ${ }^{23}$ Vitamin B12 decreases peroxide induced oxidative stress, maintains intracellular glutathione levels and inhibits caspase 3 mediated cell death (Fig. 4). ${ }^{24}$

Vitamin B12 deficiency results in increase in the levels of tumor necrosis factor- alpha (TNF- $\alpha$ ) and decrease in the levels of epidermal growth factor (EGF), which can be reversible by treatment with adequate amount of vitamin B12. These features suggest that vitamin B12 alters the function of cytokines and growth factors by modifying the activity of signaling molecules like NF- KB. ${ }^{25}$ Production of proinflammatory cytokines IL-6 and controlling NF-KB activation and progressively decreasing its levels is mediated by methyl cobalamin. GSH sparing action of vitamin B12, in turn promotes its conversion to GSSG thus generating the necessary hydrogen selenide for the production of antioxidant enzyme glutathione peroxidase (Fig. 5). It also mediates optimal phagocytic function. ${ }^{26}$ Cobalamins decrease

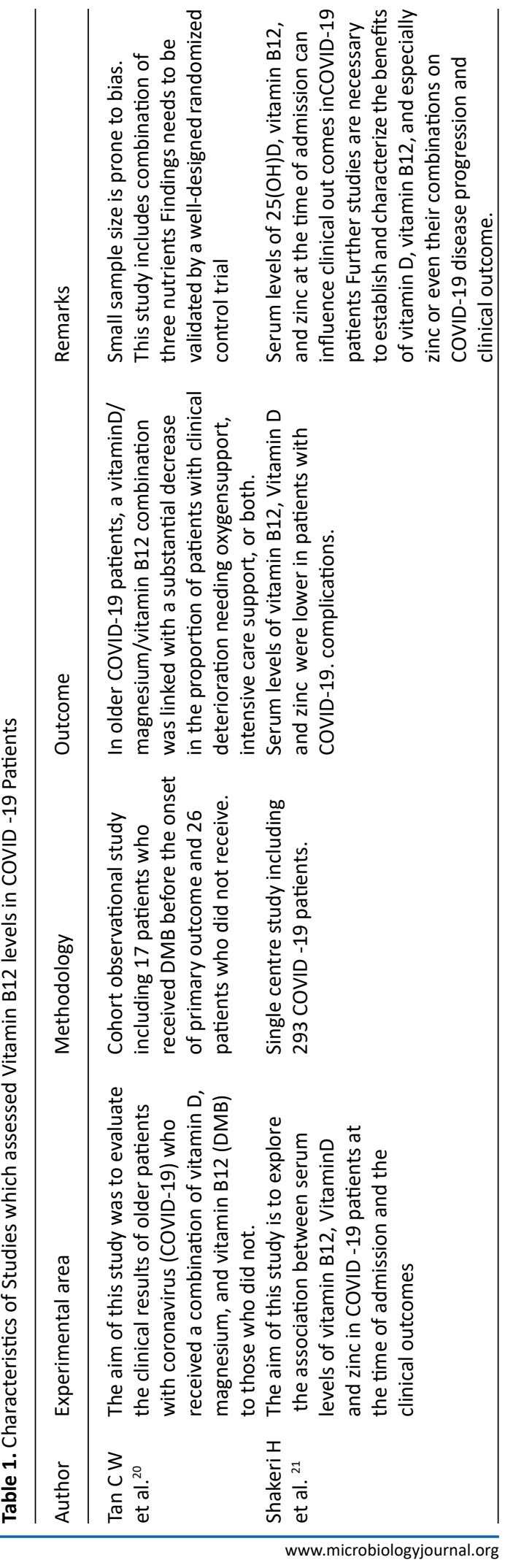


nitric oxide synthase (NOS) function and thus formation of nitric oxide (NO). Inhibition of NOS function is brought about by close approximation of cobalamin to nitric oxide synthase heme in active site. Larger the active site pocket more is its ability to inhibit NOS. ${ }^{27}$ Optimal amount of vitamin B12 is very important in maintaining host immunocompetence.

\section{Vitamin B12 Deficiency and One Carbon Metabolism}

Vitamin B12 plays a critical role in cellular and humoral immunity by mediating its action through one carbon metabolism. ${ }^{28}$ "Secondary folate deficiency" due to folate trap resulting from vitamin B12 deficiency results in decreased synthesis of thymidine and purines thus affecting the production of DNA and RNA which are important for cell division, formation of red blood cells and hence immunity. ${ }^{29}$ Secondary folate deficiency leading to methyl folate trap affecting DNA and RNA synthesis will lead to alterations in the immunoglobulin secretion leading to decreased humoral immunity. ${ }^{30}$

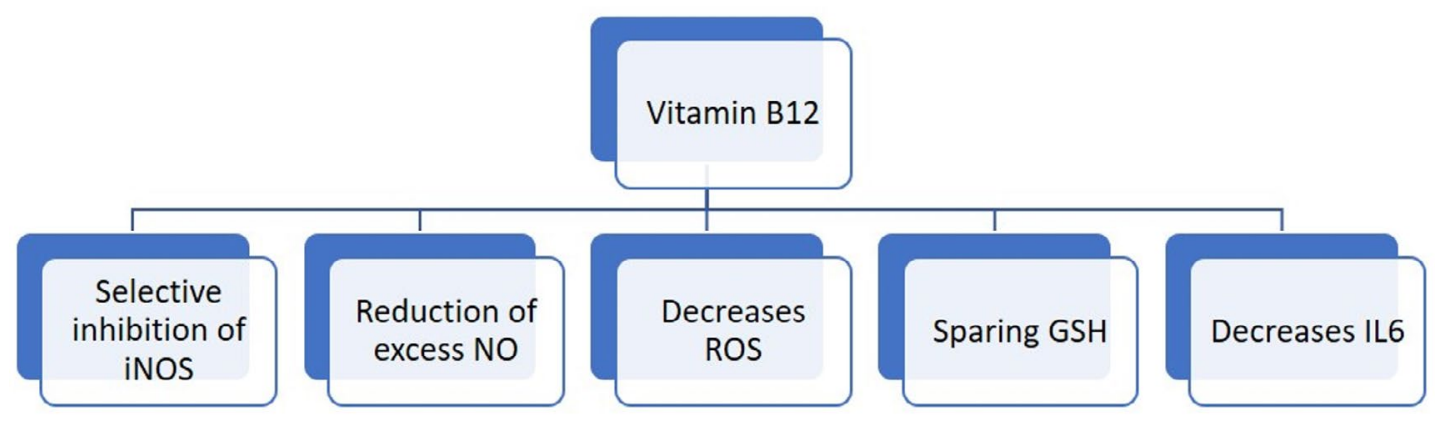

Fig. 4. Summary of Role of Vitamin B12 in Immunomodulation.

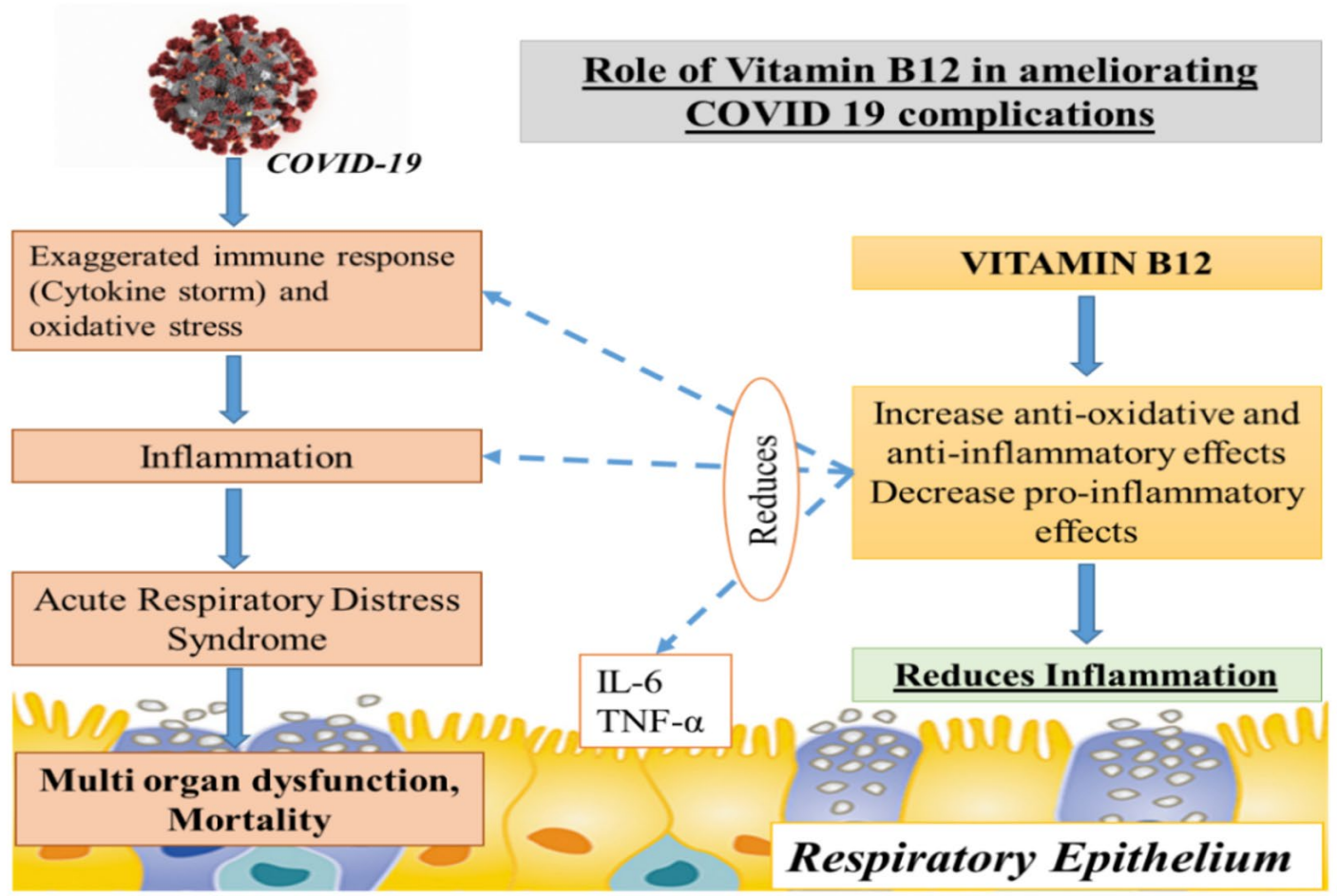

Fig. 5. Role of Vitamin B12 in Ameliorating COVID-19 Complications. 


\section{Relationship between Vitamin B12 Deficiency and Gut Microbiome}

Gut microbes require vitamin B12 for their metabolism and they compete with dietary cobalamin. Deficiency of vitamin B12 affects the microbe balance in the gut (gut dysbiosis). A leaky gut can lead to increase in the growth of bacteria and release of its toxins to the blood stream thus increasing proinflammatory cytokines. ${ }^{31}$ Zuo $\mathrm{T}$ et al. in his study showed that alterations in gut microbiome correlated with disease severity in Covid-19 patients. ${ }^{32}$ Vitamin B12 metabolism may be impaired by SARS Co-V infection, possibly impairing the microbial proliferation in the gut causing symptoms of vitamin B12 deficiency. There is an overlap among symptoms of vitamin B12 deficiency and SARS-CoV such as, increased reactive oxygen species induced organ damage, elevated homocysteine and lactate dehydrogenase concentration, activation of the coagulation cascade and vasculopathies. ${ }^{33,34}$

Obesity and Vitamin B12

Covid - 19 complications is more common in obesity. ${ }^{35}$ It is inversely associated with vitamin $\mathrm{B} 12^{36}$ and folic acid levels ${ }^{37}$ and directly associated with homocysteine levels. ${ }^{38}$

Role of Hyperhomocysteinemia Mediated By Vitamin B12 Deficiency

Vitamin B12 deficiency results in increased homocysteine levels which is shown to be proinflammatory. It mediates inflammatory transcriptional signalling in monocytes identical to that of interferon gamma. ${ }^{39} \mathrm{~A}$ study conducted by Yang Z et al. showed that hyperhomocysteinemia was associated with increased pulmonary progression on CT scan imaging. ${ }^{40}$

Angiotensin Converting Enzyme - 2 and COVID-19 Recently angiotensin converting enzyme 2 (ACE-2) has been identified as an important receptor for SARS-CoV in cell line. ${ }^{41}$ Identification of ACE-2 as a receptor for SARS -CoV has provided an important link between immunity and inflammation. Due to differences in many important amino acid of SARS-CoV receptor binding domain, SARS Cov-2 has a higher binding affinity to human ACE 2 receptor which is responsible for the increased pathogenecity of SARS -Co V 2 virus. ${ }^{42}$ Homocysteine is known to bind to the angiotensin 1 receptor (AT1R) and thus activating its downstream deleterious consequences. It binds with very high affinity to angiotensin 1 receptor and displaces angiotensin II from the receptor. ${ }^{43,44} \mathrm{Nsp}-14$ protein of SARSCoV 2 virus is a viral methyl transferase (specially guanidine $\mathrm{N}-7$ methyl transferase). ${ }^{45,46} \mathrm{~S}$ - adenosyl methionine of the host is used by this enzyme for methylation of mRNA caps, resulting in synthesis of homocysteine more than that from human methyltransferase enzyme - which is more pronounced in vitamin B12 deficiency. ${ }^{47-49}$ SARS CoV-2 disturbs the coordination between remethylation and transsulfuration reaction through S-adenosyl methionine (SAM), ${ }^{50,51}$ SAM inhibits 5, 10-methylene, THF reductase (MTHFR) and activates cystathionine beta synthase (CBS), this reaction affords a mechanism by which remethylation and transsulfuration are coordinated. This co-ordination is disturbed by SARS Co-V 2 mediated enhanced methylation demands which leads to reduction in the concentration of Sadenosyl methionine, decreased cysatathione beta synthase activity and deviation of homocysteine from synthesis of reduced GSH which is a potential intracellular antioxidant. Thus, methyl tetrahydrofolate reductase activity (MTHFR activity) and methylation reaction via folic acid and vitamin B12 will be prioritised when compared to transsulfuration and reduced glutathione synthesis during SARS CoV-2 infection. COVID-19 poses an enormous stress on one carbon metabolism by increasing the demand but impairing the synthesis of methyl groups. ${ }^{52}$

Vitamin B12 Deficiency in Elderly and Diabetics

Vitamin B12 deficiency is common in old age which is mainly attributed to decreased absorption of vitamin B12 (pernicious anaemia, atrophic gastritis), ${ }^{53}$ malnutrition, strict vegeterians ${ }^{54}$ or due to increased urinary and gut loss of vitamin $\mathrm{B} 12^{55}$ or through age-related defects in the megalin-cubulin-amnionless complex of receptors which play a pivotal role in absorption of vitamin B12. ${ }^{56,57}$

Diabetics are prone to develop vitamin B12 deficiency, since drug "metformin" which is widely used in the treatment of type 2 diabetes ${ }^{58,59}$ has been documented to produce vitamin B12 deficiency. ${ }^{60,61}$ Metformin produces disruption of calcium mediated vitamin B12 absorption thus predisposing to develop vitamin B12 deficiency. ${ }^{62}$ India is known as the diabetes capital of the world. ${ }^{63}$ 
Owing to the increased number of diabetics in India, these patients are more likely to require expensive intensive care facilities, longer duration of hospitalization and increased mortality rates due to COVID-19. Therefore, early diagnosis of vitamin B12 deficiency is of utmost importance especially in these vulnerable groups.

\section{Diagnosis of Vitamin B12}

Total serum vitamin B-12, holotranscobalamin (holo TC), methyl malonic acid and homocysteine levels are assesed for reliable evaluation of vitamin B12 status. ${ }^{64}$ Homocysteine levels can be increased in folate deficiency too, determination of plasma folate may help to differentiate between folate and vitamin B12 deficiency. ${ }^{62,63}$ Determination of S- adenosyl methionine and S- adenosyl homocysteine ratio also can be determined to assess the vitamin B12 status. ${ }^{65}$

\section{Treatment of vitamin B12}

If diagnosed treatment should be initiated to restore the adequate vitamin B12 levels. Vitamin B12 and folate in combination with glutathione forms the mainstay to treat vitamin B12 deficiency. ${ }^{66}$

This review's limitation is the presence of few clinical studies on the potential role of vitamin B12 on COVID-19. More in vivo clinical studies would enhance the evidence and could further validate the potential role of vitamin B12 in COVID-19.

\section{CONCLUSION}

Vitamin B12's antioxidant and antiinflammatory properties may help to prevent multiorgan dysfunction and therefore COVID-19 complications from progressing. Vitamin B12 insufficiency must be treated aggressively, particularly in the elderly and diabetics. Prevention of nutritional deficiencies, identification of highrisk individuals of suboptimal nutritional status and implementation of safe and effective nutritional guidelines may help in strengthening the ability of the individuals to combat COVID-19 especially in developing countries like India. Further research into the role of Vitamin B12 in COVID-19 patients may yield new insights into the most effective and well-tolerated dose and timing of Vitamin B12 delivery in COVID-19 patients.

\section{ACKNOWLEDGMENTS}

The authors are thankful to K.S. Hegde Medical Academy, Nitte (Deemed to be University), Mangalore, India for providing all the facilities to complete this work.

\section{CONFLICT OF INTEREST}

conflict of interest.

\section{AUTHORS' CONTRIBUTION}

All authors listed have made a substantial, direct and intellectual contribution to the work, and approved it for publication.

\section{FUNDING}

None.

\section{DATA AVAILABILITY}

All data generated or analyzed during this study are included in the manuscript.

\section{ETHICS STATEMENT}

Not applicable.

\section{REFERENCES}

1. Zu ZY, Jiang MD, Xu PP, et al. Coronavirus disease 2019 (COVID-19): a perspective from China. Radiology. 2020;296(2):E15-25. doi: 10.1148/radiol.2020200490

2. Gupta R, Bedi M, Goyal P, Srishti Wadhera S, and Verma V. Analysis of COVID-19 Tracking Tool in India: Case Study of Aarogya Setu Mobile Application. Digit. Gov.: Res. Pract. 2020. 1,4, Article 28: 8. doi: 10.1145/3416088

3. Shahid Z, Kalayanamitra R, McClafferty B, et al. COVID-19 and older adults: what we know. J Am Geriatr Soc. 2020;68(5):926-929. doi: 10.1111/ jgs.16472

4. Huang I, Lim MA, Pranata R. Diabetes mellitus is associated with increased mortality and severity of disease in COVID-19 pneumonia-a systematic review, meta-analysis, and meta-regression. Diabetes Metab Syndr: Clin Res Rev. 2020;14(4):395-403. doi: 10.1016/j.dsx.2020.04.018

5. Gupta R, Hussain A, Misra A. Diabetes and COVID-19: evidence, current status and unanswered research questions. Eur J Clin Nutr. 2020;74(6):864-870. doi: 10.1038/s41430-020-0652-1

6. Lippi G, Sanchis-Gomar F, Henry BM. Coronavirus disease 2019 (COVID-19): the portrait of a perfect storm. J Trans/ Med. 2020; 8(7):497. doi: 10.21037/ atm.2020.03.157

7. Maleszewski JJ, Young PM, Ackerman MJ, Halushka MK. Urgent Need for Studies of the Late Effects of SARS-CoV-2 on the Cardiovascular System. Circulation. 2021 Mar 30;143(13):1271-1273. doi: 10.1161/ 
CIRCULATIONAHA.120.051362. Epub 2020 Sep 24.

8. National Center for Biotechnology Information. PubChem Database. Cyanocobalamin, CID $=5311498$ https://pubchem.ncbi.nlm.nih.gov/compound/ Cyanocobalamin. (accessed on Apr. 16, 2020)

9. Krautler B. Vitamin B12: chemistry and biochemistry. Biochem Soc Trans. 2005;33(4):806-810. doi: 10.1042/ BST0330806

10. Andres E, Dali-Youcef N. Cobalamin (vitamin B12) malabsorption. Molecular Nutrition. 2020:367-386. doi: 10.1016/B978-0-12-811907-5.00014-2

11. Mikkelsen K, Stojanovska L, Prakash M, Apostolopoulos $V$. The effects of vitamin B on the immune/cytokine network and their involvement in depression. Maturitas. 2017;96:58-71. doi: 10.1016/j. maturitas.2016.11.012

12. Zhang M, Han W, Hu S, Xu H. Methylcobalamin: a potential vitamin of pain killer. Neural Plast. 2013;2013:424651. doi: 10.1155/2013/424651.

13. Gupta JK, Sana QS. Potential benefits of methylcobalamin: A review. Austin J Pharmacol Ther. 2015; 3(3):1076.

14. Brown KL. Chemistry and enzymology of vitamin B12. Chem Rev. 2005;105(6):2075-2149. doi: 10.1021/ cr030720z

15. Marsh EN, Melendez GD. Adenosylcobalamin enzymes: theory and experiment begin to converge. Biochim Biophys Acta. 2012;1824(11):1154-1164. doi: 10.1016/j.bbapap.2012.03.012

16. Bousquet J, Anto JM, laccarino G, et al. Is diet partly responsible for differences in COVID-19 death rates between and within countries? Clin Transl Allergy. 2020;10:16. doi: 10.1186/s13601-020-00323-0

17. Dang $Y$, Zhou T, Hao L, Cao J, Sun Y, Pan D. In vitro and in vivo stud ies on the angiotensinconverting enzyme inhibitory activity pep tides isolated from broccoli protein hydrolysate. J Agric Food Chem. 2019;67(24):6757-6756. doi: 10.1021/ acs.jafc.9b01137

18. Gharehbeglou P, Jafari SM. Antioxidant components of brassica vegeta bles including turnip and the infuence of processing and storage on their anti-oxidative properties. Curr Med Chem. 2019;26(24):4559-4572. 30. doi: $10.2174 / 0929867325666181115111040$

19. Patra JK, Das G, Paramithiotis S, Shin HS. Kimchi and other widely con sumed traditional fermented foods of Korea: a review. Front Microbiol. 2016;7:1493. doi: 10.3389/fmicb.2016.01493

20. Tan CW, Ho LP, Kalimuddin S, et al. Cohort study to evaluate effect of vitamin $D$, magnesium, and vitamin B12 in combination on severe outcome progression in older patients with coronavirus (COVID-19). Nutrition. 2020;79-80:111017. doi: 10.1016/j.nut.2020.111017

21. Shakeri H, Azimian A, Moghaddam HM, et al. Evaluation of the relationship between serum levels of zinc, Vitamin B12, vitamin D, and clinical outcomes in patients with COVID-19. J. Med virol. 2021:1-6. doi: 10.1002/jmv. 27277

22. Tamura J, Kubota K, Murakami H, et al. Immunomodulation by vitamin B12: augmentation of $\mathrm{CD}^{+} \mathrm{T}$ lymphocytes and natural killer (NK) cell activity in Vitamin B12-deficient patients by methyl-B12 treatment. Clin Exp Immunol. 1999;116(1):28-32. doi: 10.1046/j.1365-2249.1999.00870.x

23. Erkurt MA, Aydogdu I, Dikilitas M, et al. Effects of cyanocobalamin on immunity in patients with pernicious anemia. Med Princ Pract. 2008;17(2):131135. doi: 10.1159/000112967

24. Birch CS, Brasch NE, McCaddon A, Williams JH. A novel role for vitamin B12: cobalamins are intracellular antioxidants in vitro. Free Radic Biol Med. 2009;47(2):184-188. doi: 10.1016/j. freeradbiomed.2009.04.023

25. Veber D, Mutti E, Tacchini L, Gammella E, Tredici G, Scalabrino $G$. Indirect down-regulation of nuclear NF-KB levels by cobalamin in the spinal cord and liver of the rat. J Neurosci Res. 2008;86(6):1380-1387. doi: 10.1002/jnr.21599

26. Manzanares W, Hardy G. Vitamin B12 Pharmaconutrition for COVID-19. Revista de Nutrición Clinica y Metabolismo. 2021;4(1):65-69. doi: 10.35454/ rncm.v4n1.187

27. Weinberg JB, Chen $\mathrm{Y}$, Jiang $\mathrm{N}$, Beasley BE, Salerno JC, Ghosh DK. Inhibition of nitric oxide synthase by cobalamins and cobinamides. Free Radic Biol Med. 2009;46(12):1626-1632. doi: doi: 10.1016/j. freeradbiomed.2009.03.017

28. Gombart AF, Pierre A, Maggini S. A review of micronutrients and the immune system-working in harmony to reduce the risk of infection. Nutrients. 2020;12(1):236. doi: 10.3390/nu12010236

29. Saeed F, Nadeem M, Ahmed RS, Tahir Nadeem $M$, Arshad MS, Ullah A. Studying the impact of nutritional immunology underlying the modulation of immune responses by nutritional compounds - a review. Food Agric Immunol. 2016;27(2):205-229. doi: 10.1080/09540105.2015.1079600

30. Maggini S, Wintergerst ES, Beveridge S, Hornig $\mathrm{DH}$. Selected vitamins and trace elements support immune function by strengthening epithelial barriers and cellular and humoral immune responses. British J Nutr. 2007;98(S1):S29-35. doi: 10.1017/ S0007114507832971

31. Nagpal R, Mainali R, Ahmadi S, et al. Gut microbiome and aging: Physiological and mechanistic insights. Nutr Healthy Aging. 2018;4(4):267-285. doi: 10.3233/NHA170030

32. Zuo T, Zhang F, Lui GC, et al. Alterations in gut microbiota of patients with COVID-19 during time of hospitalization. Gastroenterology. 2020;159(3):944955. doi: 10.1053/j.gastro.2020.05.048

33. Sabry W, Elemary M, Burnouf T, Seghatchian J, Goubran H. Vitamin B12 deficiency and metabolismmediated thrombotic microangiopathy (MM-TMA). Transfus Apher Sci. 2020;59(1):102717. doi: 10.1016/j. transci.2019.102717

34. Grange S, Bekri S, Artaud-Macari E, et al. Adult-onset renal thrombotic microangiopathy and pulmonary arterial hypertension in cobalamin C deficiency. The Lancet. 2015;386(9997):1011-1012. doi: 10.1016/ S0140-6736(15)00076-8

35. Cariou B, Hadjadj S, Wargny M, et al. Phenotypic characteristics and prognosis of inpatients with COVID-19 and diabetes: the CORONADO study. 
Diabetologia. 2020;63(8):1500-1515. doi: 10.1007/ s00125-020-05180-x

36. Sun $Y$, Sun $M$, Liu B, et al. Inverse association between serum vitamin B12 concentration and obesity among adults in the United States. Front Endocrinol (Lausanne). 2019;10:414. doi: 10.3389/ fendo.2019.00414

37. Kim H, Hwang J-Y, Kim K-N, et al. Relationship between body-mass index and serum folate concentrations in pregnant women. Eur J Clin Nutr. 2012;66(1):136-138. doi: 10.1038/ejcn.2011.160

38. Lind MV, Lauritzen L, Vestergaard $\mathrm{H}$, et al. Onecarbon metabolism markers are associated with cardiometabolic risk factors. Nutr Metab Cardiovasc Dis. 2018;28(4):402-410. doi: 10.1016/j. numecd.2018.01.005

39. Meng S, Ciment S, Jan M, et al. Homocysteine induces inflammatory transcriptional signaling in monocytes. Front Biosci. 2013;18:685-695. doi: 10.2741/4131

40. Yang Z, Shi J, He Z, et al. Predictors for imaging progression on chest $\mathrm{CT}$ from coronavirus disease 2019 (COVID-19) patients. Aging (Albany NY). 2020;12(7):6037-6048. doi: 10.18632/aging.102999

41. Pitek AS, Wen AM, Shukla S, Steinmetz NF. The protein corona of plant virus nanoparticles influences their dispersion properties, cellular interactions, and in vivo fates. Small. 2016;12(13):1758-1769. doi: 10.1002/ smll.201502458

42. Gheblawi M, Wang K, Viveiros A, et al. Angiotensinconverting enzyme 2: SARS-CoV-2 receptor and regulator of the renin-angiotensin system: celebrating the 20th anniversary of the discovery of ACE2. Circ Res. 2020;126(10):1456-1474. doi: 10.1161/ CIRCRESAHA.120.317015

43. Verdecchia P, Cavallini C, Spanevello A, Angeli F. The pivotal link between ACE2 deficiency and SARS-CoV-2 infection. Eur J Intern Med. 2020;76:14-20. doi: 10.1016/j.ejim.2020.04.037

44. Li T, Yu B, Liu Z, et al. Homocysteine directly interacts and activates the angiotensin II type I receptor to aggravate vascular injury. Nat Commun. 2018;9(1):11. doi: 10.1038/s41467-017-02401-7

45. Singh Y, Gupta G, Kazmi I, et al. SARS CoV-2 aggravates cellular metabolism mediated complications in COVID-19 infection. Dermatologic Therapy. 2020;33(6):e13871. doi: 10.1111/dth.13871

46. Bouvet $\mathrm{M}$, Debarnot $\mathrm{C}$, Imbert I, et al. In vitro reconstitution of SARS-coronavirus mRNA cap methylation. PLoS Pathog. 2010;6(4):e1000863. doi: 10.1371/journal.ppat.1000863

47. Scott J, Weir D. The methyl folate trap: A physiological response in man to prevent methyl group deficiency in kwashiorkor (methionine deficiency) and an explanation for folic-acid-induced exacerbation of subacute combined degeneration in pernicious anaemia. The Lancet. 1981; 318(8242):337-340. doi: 10.1016/S0140-6736(81)90650-4

48. Selhub J, Miller JW. The pathogenesis of homocysteinemia: interruption of the coordinate regulation by S-adenosylmethionine of the remethylation and transsulfuration of homocysteine. Am J Clin Nutr. 1992; 55(1):131-138. doi: 10.1093/

\section{ajcn/55.1.131}

49. Jhee KH, Kruger WD. The role of cystathionine betasynthase in homocysteine metabolism. Antioxid Redox Signal. 2005;7(5-6):813-822. doi: 10.1089/ ars.2005.7.813

50. Mccaddon A, Regland B. COVID-19: A methyl group assault. Medical hypotheses. 2021:149;110543. doi: 10.1016/j.mehy.2021.110543

51. Stabler SP, Allen RH. Vitamin B12 deficiency as a worldwide problem. Annu Rev Nutr. 2004;24:299-326. doi: 10.1146/annurev.nutr.24.012003.132440

52. Hunt A, Harrington D, Robinson S. Vitamin B12 deficiency. Bmj. 2014;349. doi: 10.1136/bmj.g5226

53. Pannerec A, Migliavacca E, De Castro A, et al. Vitamin B12 deficiency and impaired expression of amnionless during aging. J Cachexia, Sarcopenia Muscle. 2018;9:41-52. doi: 10.1002/jcsm.12260

54. Christensen EI, Birn H. Megalin and cubilin: multifunctional endocytic receptors. Nat Rev Mol Cell Biol. 2002;3(4):258-267. doi: 10.1038/nrm778

55. Moestrup SK, Kozyraki R, Kristiansen M, et al. The intrinsic factor-vitamin B12 receptor and target of teratogenic antibodies is a megalin-binding peripheral membrane protein with homology to developmental proteins. J Biol Chem. 1998;273:5235-5242. doi: 10.1074/jbc.273.9.5235

56. Chamberlain JJ, Herman WH, Leal S, et al. Pharmacologic therapy for type 2 diabetes: synopsis of the 2017 American Diabetes Association Standards of Medical Care in Diabetes. Ann Intern Med. 2017;166(8):572578. doi: 10.7326/M16-2937

57. Wee AK. COVID-19's toll on the elderly and those with diabetes mellitus-Is vitamin B12 deficiency an accomplice? Medical Hypotheses. 2021;146:110374. doi: 10.1016/j.mehy.2020.110374

58. Aroda VR, Edelstein SL, Goldberg RB, et al. Long-term metformin use and vitamin B12 deficiency in the diabetes prevention program outcomes study. J Clin Endocrinol Metab. 2016;101:1754-1761. doi: 10.1210/ jc.2015-3754

59. Liu Q, Li S, Quan H, Li J. Vitamin B12 status in metformin treated patients: systematic review. PLOS ONE. 2014;9(6):e100379. doi: 10.1371/journal. pone. 0100379

60. Bauman WA, Shaw S, Jayatilleke E, Spungen AM, Herbert V. Increased intake of calcium reverses vitamin B12 malabsorption induced by metformin. Diabetes Care. 2000;23:1227-1231. doi: 10.2337/ diacare.23.9.1227

61. Gupta M, Singh R, Lehl SS. Diabetes in India: a long way to go. Int J Sci Rep. 2015;1(1):1-2. doi: 10.18203/ issn.2454-2156.IntJSciRep20150194

62. Green R, Allen LH, Bjorke-Monsen AL, et al. Vitamin B 12 deficiency. Nat Rev Dis Primers. 2017;3:17054. doi: 10.1038/nrdp.2017.54

63. Kondo $\mathrm{H}$, Osborne $\mathrm{ML}$, Kolhouse JF, et al. Nitrous oxide has multiple deleterious effects on cobalamin metabolism and causes decreases in activities of both mammalian cobalamin-dependent enzymes in rats. J Clin Invest. 1981;67(5):1270-1283. doi: 10.1172/ $\mathrm{JCl} 110155$

64. Hardlei TF, Obeid R, Herrmann W, Nexo E, Szecsi PB. 
Rai et al. | J Pure Appl Microbiol | 15(4):1735-1744 | December 2021 | https://doi.org/10.22207/JPAM.15.4.04

Cobalamin analogues in humans: a study on maternal and cord blood. PLOS ONE. 2013;8(4):e61194. doi: 10.1371/journal.pone.0061194

65. Bottiglieri T. Isocratic high performance liquid chromatographic analysis of S-adenosylmethionine and S-adenosy ihomocysteine in animal tissues: the effect of exposure to nitrous oxide. Biomed
Chromatogr. 1990;4(6):239-241. doi: 10.1002/ bmc.1130040606

66. Galmes S, Serra F, Palou A. Current state of evidence: influence of nutritional and nutrigenetic factors on immunity in the COVID-19 pandemic framework. Nutrients. 2020;12(9):2738. doi: 10.3390/nu12092738 